\title{
EMX2 is epigenetically silenced and suppresses epithelial-mesenchymal transition in human esophageal adenocarcinoma
}

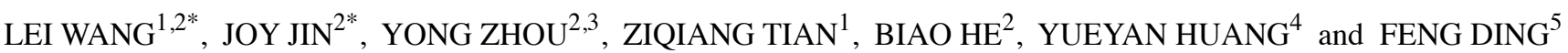 \\ ${ }^{1}$ Department of Thoracic Surgery, Fourth Hospital of Hebei Medical University, Shijiazhuang, Hebei 050011, P.R. China; \\ ${ }^{2}$ Thoracic Oncology Program, Department of Surgery, Helen Diller Family Comprehensive Cancer Center, \\ University of California, San Francisco, San Francisco, CA 94115, USA; ${ }^{3}$ Department of Respiratory Diseases, \\ Sir Run Run Shaw Hospital, Zhejiang University, Hangzhou, Zhejiang 310016; ' Department of Pharmacology, \\ Medical College of Jiaxing University, Jiaxing, Zhejiang 314000; ${ }^{5}$ Department of Biotechnology and Biomedicine, \\ Zhejiang Provincial Key Laboratory of Applied Enzymology and Jiaxing ACCB Diagnostics, \\ Yangze Delta Region Institute of Tsinghua University Zhejiang, Jiaxing, Zhejiang 314006, P.R. China
}

Received February 16, 2019; Accepted July 23, 2019

DOI: $10.3892 /$ or.2019.7284

\begin{abstract}
Esophageal adenocarcinoma (EAC) is an aggressive and challenging disease to treat, with an overall five-year survival rate of $<20 \%$. Early malignant cell dissemination contributes to this poor prognosis. Epithelial-mesenchymal transition (EMT) induces the invasion and metastasis of carcinoma cells. Empty spiracles homeobox 2 (EMX2) is a homeodomain-containing transcription factor, which is associated with numerous cancer types, and has been demonstrated to regulate EMT. In the present study, 48 pairs of EAC and adjacent normal tissues were analyzed. The results revealed that EMX2 was downregulated in EAC tissues, and its expression was negatively correlated with the DNA hypermethylation of its promoter. Additionally, the OE19 and OE33
\end{abstract}

Correspondence to: Dr Yueyan Huang, Department of Pharmacology, Medical College of Jiaxing University, Jiaxing, Zhejiang 314000, P.R. China

E-mail: hyylinda@163.com

Dr Feng Ding, Department of Biotechnology and Biomedicine, Zhejiang Provincial Key Laboratory of Applied Enzymology, Yangze Delta Region Institute of Tsinghua University Zhejiang and ACCB Diagnostics, Jiaxing, Zhejiang 314006, P.R. China

E-mail: fding7@foxmail.com

*Contributed equally

Abbreviations: DAC, 5-aza-2'-deoxycytidine; EAC, esophageal adenocarcinoma; EMT, epithelial-mesenchymal transition; N, normal; p, phosphorylated; RT-qPCR, reverse transcription-quantitative PCR; qMSP, quantitative methylation-specific polymerase chain reaction; T, tumor; UCSF, University of California San Francisco

Key words: empty spiracles homeobox 2, epithelial-mesenchymal transition, esophageal adenocarcinoma
EAC cell lines were treated with the DNA methyltransferase inhibitor 5-aza-2'-deoxycytidine, and the results indicated that EMX2 expression was increased. Overexpressing EMX2 in EAC cell lines enhanced the expression of apoptotic markers, inhibited cell migration and invasion, led to the upregulation of E-cadherin and the downregulation of mesenchymal markers, and suppressed AKT, mTOR and S6K phosphorylation. Furthermore, EMX2 overexpression sensitized EAC cells to cisplatin. These results demonstrated that EMX2 inhibited the AKT/mTOR/S6K signaling pathway and decreased EMT. However, the downregulation of EMX2 was revealed to be associated with EMT in EAC, indicating that EMX2 may be a potential target for the management of EAC.

\section{Introduction}

Worldwide, esophageal cancer is the eighth most common cancer, but the sixth most common cause of cancer-associated deaths (1). Esophageal cancer is comprised of two pathologically and epidemiologically distinct subtypes: Esophageal adenocarcinoma (EAC) and esophageal squamous carcinoma (ESCC). EAC resembles a chromosomally unstable variant of gastric adenocarcinoma, whereas ESCC shares many characteristics with squamous cell carcinoma of the head and neck (2). Although ESCC is the most prevalent subtype worldwide, EAC incidence rates have risen in developed countries over the past four decades (3). The majority of patient admissions exhibit locally advanced or metastatic disease, and as a result of this late diagnosis, EAC is an aggressive and difficult disease to treat, with an overall five-year survival rate of $<20 \%$ (4). Early malignant tumor cell dissemination contributes to the poor prognosis of EAC (5). Therefore, there is an urgent requirement for research into the mechanisms of EAC tumor invasion and metastasis. With the information gained from these studies, novel treatment options can be explored.

Epithelial-mesenchymal transition (EMT) is a process that induces the invasion and metastasis of epithelial cancer cells (6-9). 
During EMT, epithelial cells lose their apical-basal polarity and intercellular adhesion, and gain mesenchymal cell characteristics, including increased migratory activity and fibroblast-like morphology. EMT is modulated by changes at the genomic, epigenomic, transcriptomic and proteomic levels (6-9). In previous studies investigating EMT in EAC, the downregulation of epithelial markers, and the upregulation of mesenchymal markers with concomitant transforming growth factor (TGF)- $\beta 1$, were observed in invasive margins compared with the central tumor (10). The signaling of bone morphogenetic protein 4 (BMP4), a TGF-family protein, has been revealed to induce the EMT-like phenotype in EAC cell lines (11). Additionally, a recent study has demonstrated that AKT inhibition reduces EMT, whereas glioma-associated oncogene (GLI) activation promotes EMT in EAC (12). Furthermore, the overexpression of EMT-promoting transcription factors, including SLUG (13) and snail family transcriptional repressor 2 (SNAL2) (14) have been associated with poor EAC prognosis. Despite these recent research advances, the process of EMT in EAC remains poorly understood.

Empty spiracles homeobox 2 (EMX2) is a member of the Homeobox gene family, which is comprised of a large group of developmental regulators that are vital for growth and differentiation (15). EMX2 serves important roles in cortical development (16), neurogenesis $(17,18)$, early hair cell development (19), the stereociliary bundle orientation of sensory hair cells (20) and mammalian reproduction (21). Recent studies have indicated the potential involvement of EMX2 in various different human cancer types, including lung (22-25), gastric (26), endometrial (27) and colorectal cancer $(28,29)$. It has also been revealed that EMX2 inhibits the proliferation of lung (23), gastric (26) and colorectal (29) cancer cells, and suppresses the invasive phenotype of lung (23) and colorectal $(28,29)$ cancer cells. These results indicate a possible role of EMX2 in the process of EMT. In the present study, EMX2 expression was determined in EAC tissues and cell lines, and the role of EMX2 in the EMT of EAC was investigated. The results demonstrated that the downregulation of EMX2 was associated with EMT in EAC, and that EMX2 may suppress EMT via the inhibition of AKT signaling in the AKT/mTOR/S6K pathway.

\section{Materials and methods}

Tissue specimens. Tissue specimens were collected between March 2015 and February 2016 from 48 patients (age, 67.5 \pm 10.6 ; males 29; females 19) who underwent surgical resection for EAC at the University of California San Francisco Thoracic Oncology Program (Table SI). Tissue samples were snap-frozen in liquid nitrogen immediately after resection and stored at $-170^{\circ} \mathrm{C}$ prior to use. The present study was conducted in accordance with the ethical standards of the Declaration of Helsinki, as well as national and international guidelines, with the approval by the institutional review board of the University of California, San Francisco (UCSF). Studies involving patient tissues were approved by the Committee on Human Research (CHR approval number: H8714-11647-10) at UCSF, and written, informed consent was obtained for each patient prior to tissue specimen collection.

Protein extraction and western blot analysis. Total protein was extracted from OE19 and OE33 cells using M-PER Mammalian
Protein Extraction Reagent (Thermo Fisher Scientific, Inc.) and Complete Protease Inhibitor Cocktails (Roche Diagnostics), according to the manufacturer's protocol. Protein concentrations were determined using a Pierce BCA Protein Assay kit (Thermo Fisher Scientific, Inc.), and $10 \mu \mathrm{g}$ protein/lane was separated using SDS-PAGE on a 4-20\% gel, prior to transfer to Immobilon-P membranes (EMD Millipore). The membranes were then blocked in 5\% non-fat milk and incubated with primary antibodies overnight at $4^{\circ} \mathrm{C}$. The membranes were then incubated with the appropriate secondary antibodies (goat anti-rabbit IgG HRP conjugate 31460 at 1:20,000 or goat anti-mouse IgG HRP conjugate 31430 at 1:10,000; Thermo Fisher Scientific, Inc.), and signals were detected using an ECL blotting analysis system. The primary antibodies used were as follows: Cyclin D1 (cat. no. sc-8396 at 1:200 dilution), B-cell lymphoma 2 (BCL2; cat. no. sc-7382 at 1:200 dilution ), and fas cell surface death receptor (FAS; cat. no. sc-8009 at 1:500 dilution; all from Santa Cruz Biotechnology, Inc.); phosphorylated (p) m-TOR (cat. no. ab1093; 1:200 dilution) and p-S6K1 (cat. no. ab131436; 1:250 dilution) (both from Abcam); N-cadherin (cat. no. 05-915; 1:200 dilution), E-cadherin (cat. no. 07-697; 1:100 dilution) and $\beta$-catenin (cat. no. 04-958; 1:200 dilution; all from Sigma-Aldrich, Merck KGaA); vimentin (cat. no. 3932; 1:200 dilution), and p-AKT (cat. no. 9271; 1:200 dilution; both Cell Signaling Technology, Inc.); GAPDH (cat. no. sc32233; 1:500 dilution; Santa Cruz Biotechnology, Inc.) and EMX2 (cat. no. PA5-84687; 1:400 dilution; Thermo Fisher Scientific, Inc.).

Cell culture and transfection. Human EAC cell lines OE19 and OE33 were purchased from the American Type Culture Collection and cultured in RPMI-1640 medium (Thermo Fisher Scientific, Inc.). Cells were supplemented with $10 \%$ FBS and penicillin/streptomycin $(100 \mathrm{mg} / \mathrm{ml})$ at $37^{\circ} \mathrm{C}$ in a humidified $5 \% \mathrm{CO}_{2}$ incubator. To establish cell lines exhibiting EMX2 overexpression, cultured OE19 and OE33 cells were transfected with empty pCDNA3.1 vector controls or with pCDNA3.1 vectors containing EMX2. Transfection was performed using Lipofectamine ${ }^{\circledR} 2000$ (Thermo Fisher Scientific, Inc.).

Treatment with 5-aza-2'-deoxycytidine (DAC). To analyze the restoration of EMX2 gene expression, cells were treated with $5 \mu \mathrm{M}$ DAC (Sigma-Aldrich; Merck KGaA) for 4 days, as previously described (23). Medium was replaced daily with fresh medium containing the same dose of DAC. Control cells were cultured with fresh medium containing DMSO. Cells were subsequently isolated for further analysis using DNA and RNA extraction.

Quantitative methylation-specific PCR (qMSP). Formalin-fixed, paraffin-embedded tissues were de-paraffinized in xylene (Sigma-Aldrich; Merck KGaA) and rehydrated in graded ethanol prior to bisulfite treatment, according to the manufacturer's protocol. Bisulfite conversion from tissues and cells was performed, without the prerequisite DNA purification step, using an EZ DNA Methylation-Direct kit (Zymo Research Corp.). Genomic DNA was extracted using Qiagen DNeasy kits (Qiagen, Inc.). qMSP was performed using the ABsolute Blue qPCR SYBR-Green ROX Mix (Thermo Fisher 
Scientific, Inc.) with MSP primers, which recognized either methylated or unmethylated EMX2 promoter sequences after bisulfite treatment. PCR conditions were as follows: $10 \mathrm{~min}$ at $95^{\circ} \mathrm{C} ; 40$ cycles of $30 \mathrm{sec}$ at $95^{\circ} \mathrm{C}, 30 \mathrm{sec}$ at $50^{\circ} \mathrm{C}$ and $30 \mathrm{sec}$ at $72^{\circ} \mathrm{C}$; with a final $7 \mathrm{~min}$ extension at $72^{\circ} \mathrm{C}$. Primers were designed and purchased from Eurofins MWG Operon, Inc. Sequences are as follows: ATCB forward, 5'-TGGTGATGG AGGAGGTTTAGTAAGT-3' and reverse, 5'-AACCAATAA AACCTACTCCTCCCTTAA-3'; MSP-M forward, 5'-TAG TTTTTTGTTCGTTTCGCGTTTC-3' and reverse, 5'-GAA TTAAAATAA ACGCCCCTACCGAC-3'; and MSP-U forward, 5'-GTTTTTTGTTTGTTTTGTGTTTTGA-3' and reverse, 5'-CCAAATTAAAATAAACACCCCTACCAAC-3'.

All qMSP assays were performed in triplicate using the ABI 7900HT Fast Real-Time PCR System (Applied Biosystems; Thermo Fisher Scientific, Inc.). Relative EMX2 methylation levels were determined using the $2^{-\Delta \Delta C q}$ method (30) and normalized to the housekeeping gene, ACTB, prior to calculating the ratio of tumor/paired normal tissue per patient.

$R N A$ extraction and reverse transcription-quantitative $P C R$ $(R T-q P C R)$. Total RNA was isolated using the Qiagen RNeasy kit, and cDNA was transcribed from 500 ng RNA using the iScript cDNA Synthesis kit (Bio-Rad Laboratories, Inc.) according to the manufacturer's protocol. EMX2, Aurora Kinase (AURK)A, AURKB, caspase (CASP)-3 and GAPDH mRNA expression were subsequently examined using RT-qPCR with commercially available primers and probes (Thermo Fisher Scientific, Inc.) and Relative Quantification Software (Applied Biosystems; Thermo Fisher Scientific, Inc.). Gene expression was normalized to GAPDH expression using the $2^{-\Delta \Delta C q}$ method (30).

Wound healing, Transwell invasion and cell survival assays. In the wound healing migration assays, four scratch wounds were made in each treatment condition and cell migration was determined using the average differences in distance between 0-24 h. Transwell invasion assays (Thermo Fisher Scientific, Inc.) were performed using six-well plates, according to the manufacturer's protocol. EAC cells were placed on the upper layer of a cell culture insert (Matrigel-coated membrane), and incubated for $24 \mathrm{~h}$. The migrated cells were then fixed with methanol and stained with crystal violet. For each well, three representative fields of view were selected to measure the invasion level.

To evaluate cell survival after chemotoxic treatment, cells were plated in 96-well plates at a density of 500-1,000 cells/well. The medium was changed daily. Logarithmically growing cells were treated with $5 \mu \mathrm{M}$ cisplatin or DMSO (vehicle control) for 24-72 h. Cell survival was assessed using CellTiter-Glo Luminescent Cell Viability Assay reagent (Promega Corporation), according to the manufacturer's protocol. Luminescence was measured using a GloMax-96 Microplate Luminometer (Promega Corporation). The percentage cell survival was calculated based on the measurement of untreated cells as 100\% using GraphPad Prism 6.0 software (GraphPad Software, Inc.), which was also used to generate dose-response curves and $\mathrm{IC}_{50}$ values. All experiments were conducted in triplicate and data were standardized to DMSO-treated cells.
Statistical analysis. Two-tailed Student's t-tests were performed for wound healing (migration) and Transwell (invasion) assay analyses. ANOVA and Scheffe tests were used to determine significance in luciferase reporter and qPCR data. $\mathrm{P}<0.05$ was considered to indicate a statistically significant difference.

\section{Results}

EMX2 expression is downregulated by methylation in the tissues of patients with EAC, and EAC cell lines. To determine whether EMX2 served a role in the progression of EAC, EMX2 protein expression was assessed in the EAC tissues and paired adjacent normal tissues of 48 patients with EAC, using western blot analysis. The results demonstrated that $79.2 \%$ (38/48) of tumor samples exhibited lower EMX2 protein expression compared with paired normal tissue expression (Fig. 1A and Fig. S1).

Homeobox (HOX) gene silencing by promoter methylation has been previously reported for HOXA5 and HOXA9 in breast cancer (31) and testicular germ cell tumors (32), respectively. Previous studies have revealed that the downregulation of EMX2 is associated with the hypermethylation of its promoter in lung (23) and gastric cancer (26). To determine whether promoter hypermethylation was responsible for the downregulation of EMX2 in EAC tissues, the EMX2 promoter methylation status was determined using qMSP (33). Decreased EMX2 protein expression in EAC tissues was revealed to be associated with hypermethylation of the EMX2 promoter in EAC when compared with adjacent normal tissues (Fig. 1A).

EMX2 expression in two EAC cell lines, OE19 and OE33, was also determined. When treated with the methyltransferase inhibitor, DAC, the methylation levels of EMX2 promoter decreased in OE19 and OE33 cells (Fig. 1B), and the expression of EMX2 transcripts was activated (Fig. 1C). These data indicated that EMX2 expression is downregulated by promoter hypermethylation in EAC tissues and cell lines.

EMX2 induces apoptotic marker expression in EAC cells. Genes or proteins that are associated with apoptosis or cell cycle regulation, in OE19 and OE33 cells, were assessed to determine the biological function of EMX2 in EAC progression. EMX2 expression was induced via transient transfection with a EMX2 vector, and pCDNA3.1 was used as a control, as previously described (23). mRNA expression, measured using RT-qPCR, indicated that EMX2 expression increased by 4-5 orders of magnitude subsequent to transfection with an EMX2 expression vector ( $\mathrm{P}<0.01$; Fig. $2 \mathrm{~A})$.

AURKA and AURKB are essential regulators for mitosis, and have been demonstrated to inhibit apoptosis (34-36). In the present study, EMX2 expression significantly inhibited AURKA and AURKB expression in OE19 (63\% reduction; $\mathrm{P}<0.01$; Fig. 2B) and OE33 (40\% reduction; P $<0.01$; Fig. 2B) cell lines. AURKA and AURKB protein expression also decreased following EMX2 expression (Fig. 2D). CASP3, an apoptosis marker, was upregulated following EMX2 expression and exhibited a 1.5- and 2-fold upregulation in OE19 and OE33 cells, respectively ( $\mathrm{P}<0.01$; Fig. $2 \mathrm{C}$ ). Furthermore, regulators of apoptosis and cell cycle progression, including 
A
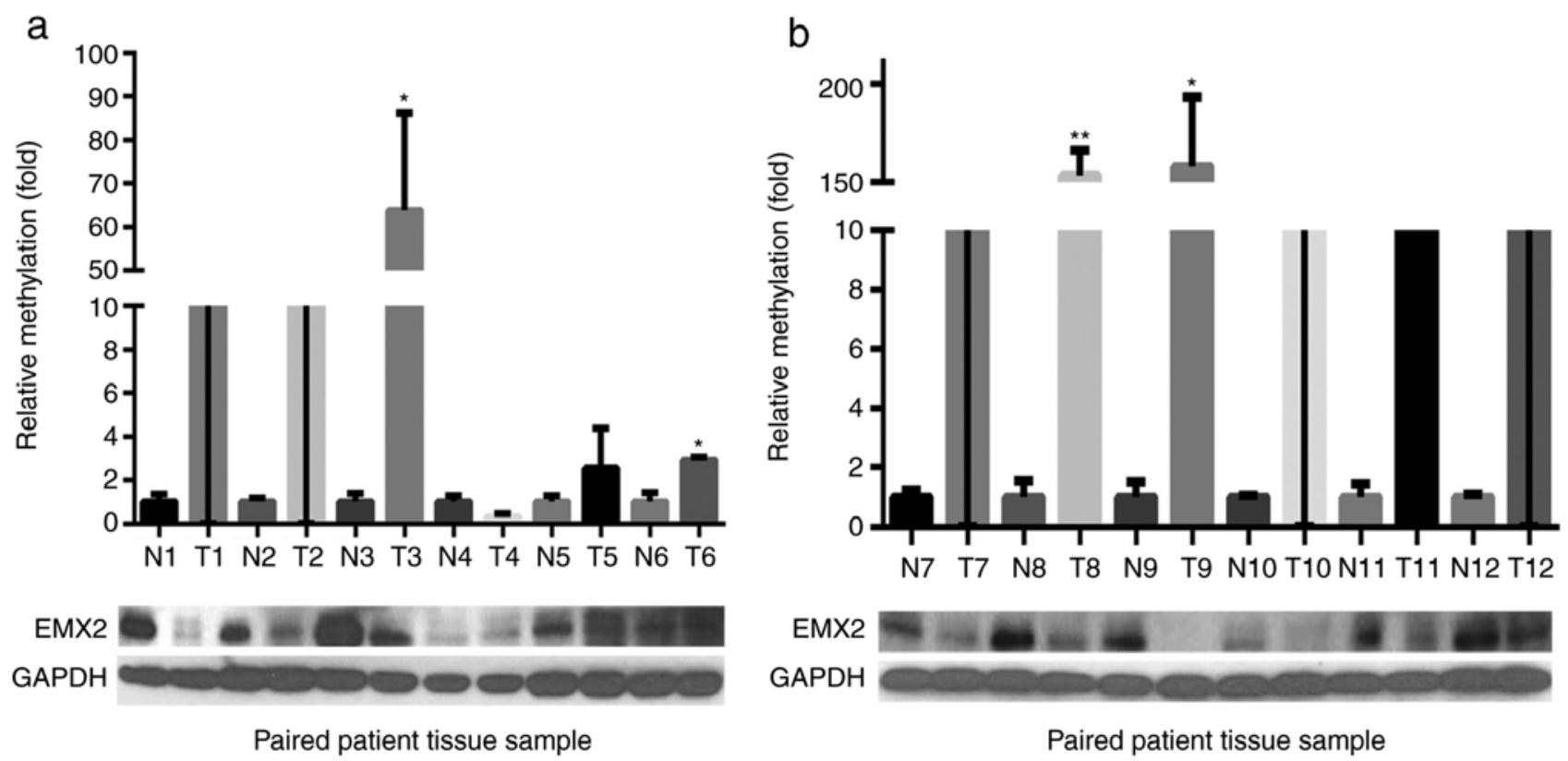

Paired patient tissue sample

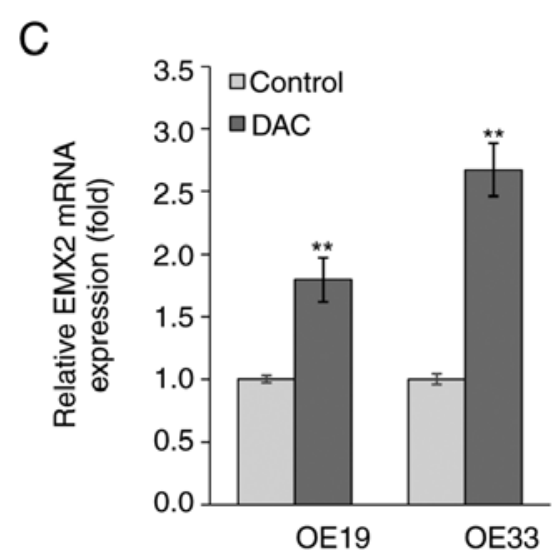

Figure 1. EMX2 expression is downregulated by methylation in EAC tissues and cell lines. (A) Western blot analysis of EMX2 and qMSP in 12 matched pairs of $\mathrm{N}$ and $\mathrm{T}$ tissues. Samples are presented with corresponding EMX2 protein levels. qMSP results were normalized to paired normal tissues, and GAPDH was used as an internal control for EMX2 protein expression. (a) $\mathrm{N}$ and $\mathrm{T}$ pairs 1-6, with values of $\mathrm{T} 1$ and $\mathrm{T} 2$ in the broken region of the $\mathrm{y}$-axis. (b) $\mathrm{N}$ and $\mathrm{T}$ pairs 7-12, with values of T7, T10, T11 and T12 in the broken region of the y-axis. (B) DAC treatment reduced EMX2 promoter methylation in OE19 and OE33 cells. EAC cells were treated with $5 \mu \mathrm{M}$ DAC, followed by qMSP analysis of EMX2 promoter methylation using reactions specific to methylated (Me) or unmethylated (U) targets. (C) DAC treatment restored EMX2 expression in OE19 and OE33 cells. The EAC cells were treated with $5 \mu$ M DAC, followed by reverse transcription-quantitative PCR examination of EMX2 mRNA expression. ${ }^{*} \mathrm{P}<0.05$ and ${ }^{* *} \mathrm{P}<0.01$. EMX2, empty spiracles homeobox 2; EAC, esophageal adenocarcinoma; qMSP, quantitative methylation-specific polymerase chain reaction; N, normal; T, tumor; DAC, 5-aza-2'-deoxycytidine.

BCL2 $(37,38)$, cyclin D1 $(39,40)$ and FAS $(41,42)$ were examined using western blot analysis (Fig. 2D). The expression of BLC2, a cell death suppressor, and cyclin D1, a protein required for G1 phase progression in the cell cycle, were negatively correlated with EMX2 expression. In contrast, the expression of FAS, which can induce apoptosis, was positively associated with EMX2. These results indicated that EMX2 promotes apoptosis and inhibits cell cycle progression in EAC.

EMX2 attenuates EAC cell migration and invasion. To determine the role of EMX2 in EAC cell migration and invasion, wound healing and Transwell invasion assays were performed in OE19 and OE33 cells. Over a period of 24 h, OE19 and OE33 cells exhibited significantly slower migration in cells transfected with EMX2 (Fig. 3A and B). Statistical analysis indicated that the migration of OE19-EMX2 and OE33-EMX2 cells was $\sim 40 \%$ of the migration exhibited by the vector control cells $(\mathrm{P}<0.01)$. Furthermore, in the Transwell invasion assays, OE19 and OE33 cells transfected with EMX2 demonstrated decreased invasion of $>50 \%$ compared with cells transfected with the vector control ( $\mathrm{P}<0.01$; Fig. $3 \mathrm{C}$ and $\mathrm{D})$.

EMX2 inhibits EMT marker expression in EAC. An important hallmark of EMT is the loss of E-cadherin expression, a cell-to-cell adhesion molecule that is encoded by $\mathrm{CDH}$, a tumor suppressor gene. It has been previously revealed that E-cadherin expression is reduced in EAC, and this decreased expression is associated with an increased frequency of lymph node metastasis and decreased patient survival (43). A previous study indicated that E-cadherin protein expression was reduced in the tumor tissues of patients with EAC. In contrast, two mesenchymal markers, $\mathrm{N}$-cadherin and 

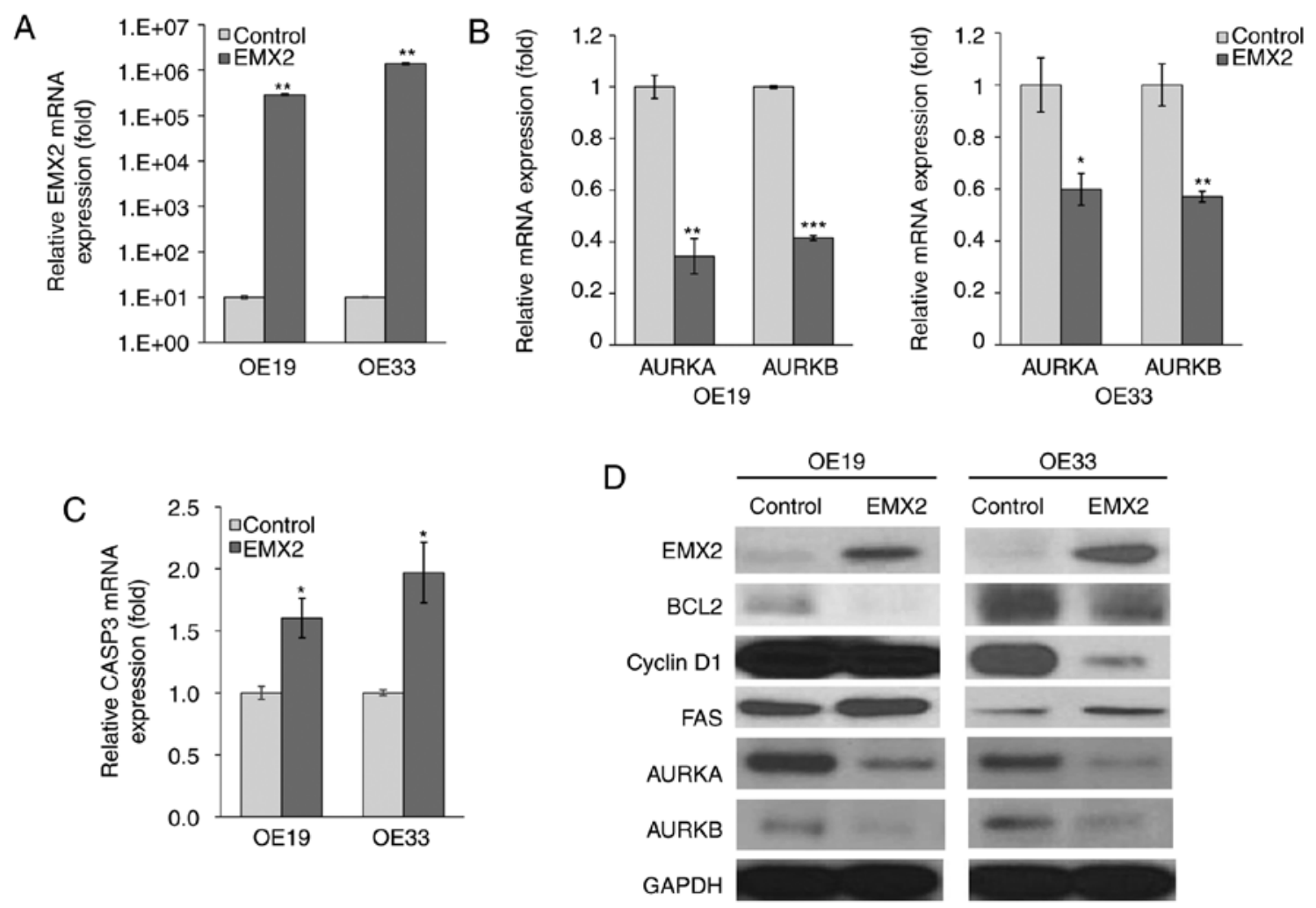

Figure 2. EMX2 induces apoptotic marker expression in EAC cells. (A) mRNA expression of EMX2 in two EAC cell lines, OE19 and OE33, that were transfected with an empty pCDNA3.1 vector control or EMX2, as determined using RT-qPCR. (B) mRNA expression of AURKA and AURKB in OE19 and OE33 cells transfected with an empty pCDNA3.1 vector control or EMX2, as determined using RT-qPCR. (C) CASP3 mRNA expression in OE19 and OE33 cells transfected with an empty pCDNA3.1 vector control or EMX2, as determined using RT-qPCR. (D) Western blot analysis of key cell cycle and apoptosis markers (BCL2, cyclin D1 and FAS), and AURKA and AURKB protein expression in OE19 and OE33 cells transfected with an empty pCDNA3.1 vector control or EMX2. GAPDH was used as a loading control. ${ }^{*} \mathrm{P}<0.05,{ }^{* *} \mathrm{P}<0.01$ and ${ }^{* * * *} \mathrm{P}<0.001$. EMX2, empty spiracles homeobox 2; EAC, esophageal adenocarcinoma; RT-q, reverse transcription-quantitative; AURK, aurora kinase; CASP3, caspase-3; BCL2, B-cell lymphoma 2; FAS, fas cell surface death receptor.

vimentin, and cell adhesion and signaling molecule, $\beta$-catenin, were overexpressed (12). Due to the fact that cell migration and invasion have been associated with altered levels of EMT biomarkers, the effect of EMX2 expression on epithelial features of EAC cells was assessed in the present study. The expression of epithelial markers, including E-cadherin, and of mesenchymal markers, including $\mathrm{N}$-cadherin and vimentin, were examined. As presented in Fig. 3E, EMX2 expression in OE19 and OE33 cells increased E-cadherin expression and decreased $\mathrm{N}$-cadherin, vimentin and $\beta$-catenin expression. These results indicated that EMX2 suppresses EMT in EAC.

EMX2 inhibits EMT by suppressing AKT/mTOR/S6K1 signaling in EAC. The present study also investigated the mechanism by which EMX2 inhibits EMT in EAC. AKT genes are overexpressed in EAC, and their expression has been associated with the pathologic response of EAC treated with neoadjuvant therapy (44). A previous study revealed the upregulation of the AKT pathway proteins p-AKT, p-mTOR and p-S6K1 in the tumor tissues of patients with EAC (12). The results of the present study demonstrated that the upregulation of AKT pathway markers was correlated with EMX2 downregulation in EAC tumor tissues (Fig. 4A). These results prompted the hypothesis that EMX2 inhibits EMT by suppressing the AKT signaling cascade. In OE19 and OE33 cells, EMX2 overexpression decreased p-MTOR, p-AKT and p-S6K1 levels (Fig. 4B). These findings indicated that EMX2 inhibits EMT in EAC cells, at least in part, by suppressing the AKT/mTOR/S6K1 signaling pathway.

EMX2 sensitizes EAC cells to cisplatin. It has been revealed that EMT serves a critical role in drug resistance. Therefore, in the present study, whether EMX2 overexpression could sensitize EAC cells to cisplatin, a widely used chemotherapy agent for EAC, was assessed (45). A cell viability assay revealed that OE19 and OE33 cells transfected with a control vector were resistant to cisplatin treatment. In contrast, when treated with cisplatin, the viability of EMX2-overexpressed OE19 and $\mathrm{OE} 33$ cells was reduced by at least $30 \%$ compared with cells transfected with a vector control (Fig. 5A). EMX2 protein expression was further confirmed using western blot analysis (Fig. 5B). These results indicated that EMX2 overexpression can sensitize EAC cells to cisplatin.

\section{Discussion}

Despite recent advances in cancer research, the survival rates of patients with EAC remain low, and EAC continues to be a poorly understood disease. EMT is an important mechanism in epithelial cancer cell invasion and metastasis, with local invasion and metastasis occurring early in the pathogenesis of EAC. Although EMT has been indicated to serve a role in the early metastasis of EAC, the regulatory mechanisms of EMT in EAC remain undetermined. 
A

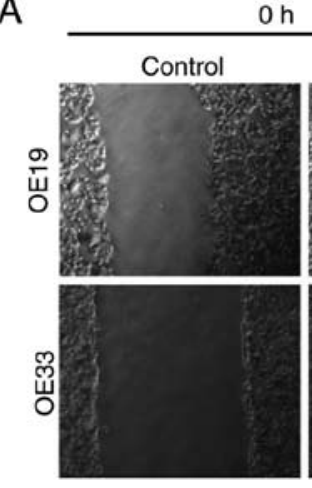
$\mathrm{h}$
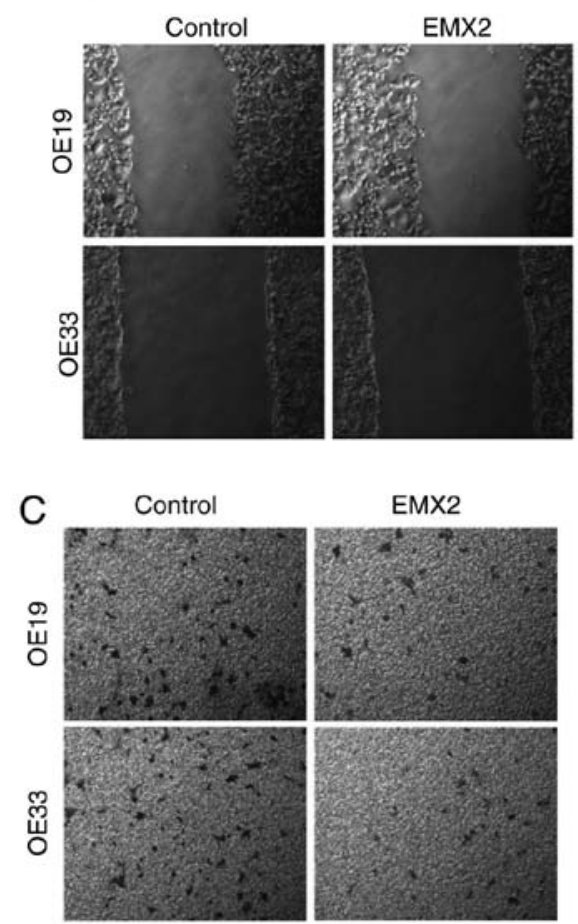

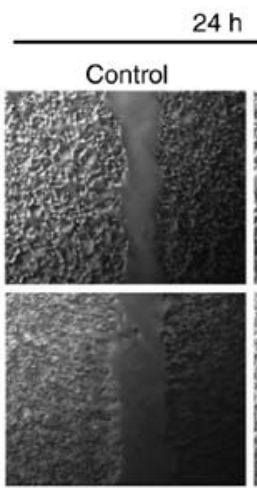
$4 \mathrm{~h}$

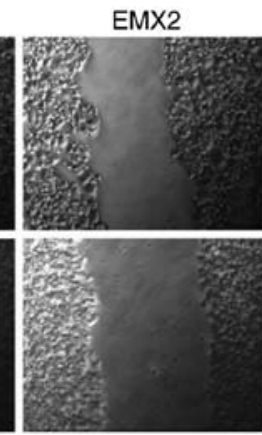

B
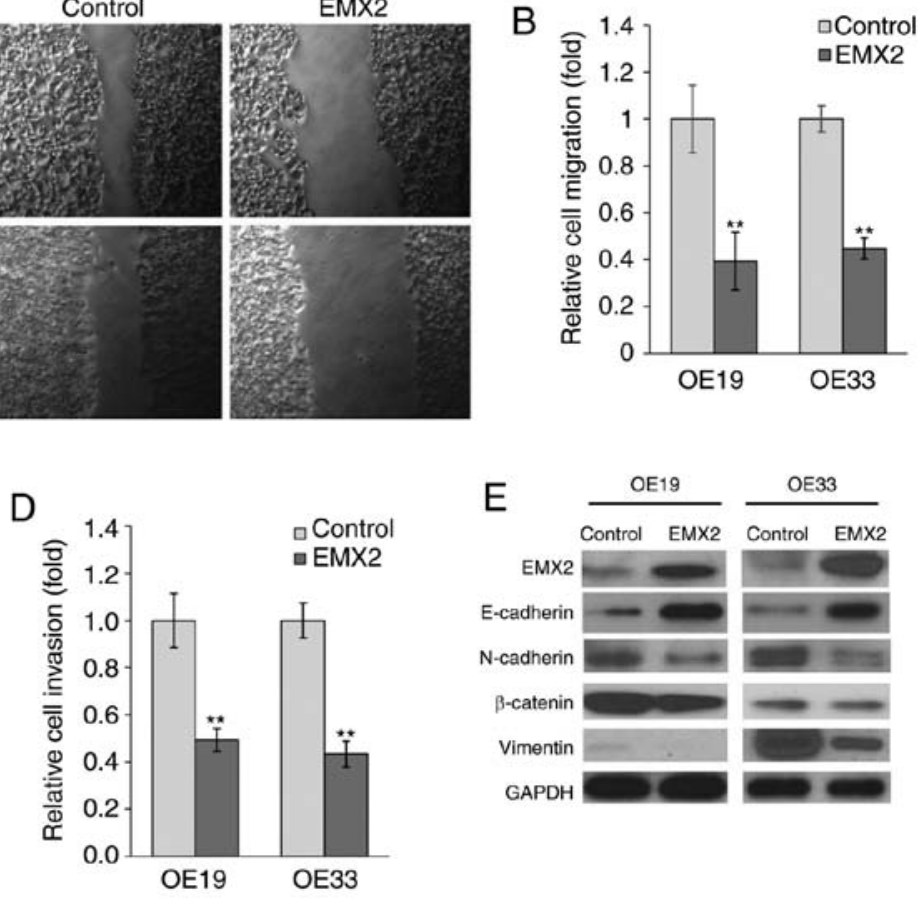

Figure 3. EMX2 reduces cell migration and invasion and EMT marker expression in EAC cells. (A) Wound healing assay of OE19 and OE33 cells transfected with EMX2 or a vector control. Representative images from 0-24 h, captured using a light microscope (x100). (B) Quantification of the migration of EAC cells after $24 \mathrm{~h}$. Results are expressed as the fold change compared with the control group. (C) Transwell invasion assay of OE19 and OE33 cells transfected with a control vector or EMX2. Representative images, captured using a light microscope at 0-24 h (x100). (D) Quantification of the invasion of EAC cells. Results are expressed as the fold change compared with the control transfection group. (E) Western blot analysis of EMT markers (N-cadherin, E-cadherin, $\beta$-catenin and vimentin) in OE19 and OE33 cells transfected with EMX2 or a vector control. GAPDH was used as a loading control. ${ }^{* *} \mathrm{P}<0.01$. EMX2, empty spiracles homeobox 2; EMT, epithelial-mesenchymal transition; EAC, esophageal adenocarcinoma.

The results of the present study demonstrated, to the best of our knowledge, for the first time, that EMX2 suppresses EMT in EAC. EMX2 is downregulated in a variety of cancer types and has been revealed to be associated with the EMT process. In a previous study, it was revealed that the overexpression of EMX2 in lung squamous cell carcinoma cells inhibited cell migration and downregulated EMT markers, whereas the knockdown of endogenous EMX2 promoted cell migration and upregulated EMT markers (25). Zhang et al (29) demonstrated that the overexpression of EMX2 significantly inhibited cell migration and invasion, and upregulated EMT marker expression, in a human colorectal cancer cell line. The present study revealed that EMX2 is downregulated in EAC tissues and cell lines, and the restoration of EMX2 in EAC cell lines induced the expression of apoptotic markers, inhibited cell migration and invasion and induced E-cadherin expression while inhibiting mesenchymal marker expression. These data indicated that EMX2 suppresses EMT in EAC.

In a previous study with lung squamous cell carcinoma (25), 12 cell lines were screened and the majority exhibited low EMX2 expression. Only two cell lines had high EMX2 expression and were used for silencing experiments. For EAC, the available cell lines were limited and none of the tested cell lines exhibited a high level of EMX2 expression. As revealed in Fig. S1, the esophageal adenocarcinomas had much lower EMX2 expression compared with the adjacent normal tissues, and some were below the detection limit. The two cell lines used in this study, OE19 and OE33, had barely detectable EMX2 (Fig. 2A and 2D). Due to the intrinsic low level of EMX2 expression, the silencing experiment was infeasible.

To illustrate the relationship between EMX2 promoter methylation and gene expression, the methylation levels of EMX2 in OE19 and OE33 cells with or without 5-aza-2'-deoxycytidine (DAC) treatment were measured. The promoter regions of EMX2 in OE19 and OE33 cells were highly methylated, which revealed decreased methylation upon DAC treatment (Fig. 1B). Consequently, EMX2 expression was increased (Fig. 1C). These results demonstrated that the downregulation of EMX2 in EAC tissues is correlated with hypermethylation of its promoter, and suggested that epigenetic silencing is an important mechanism for the downregulation of EMX2 in EAC. It corresponds with previous findings regarding epigenetic silencing of EMX2 in lung (23) and gastric cancer (26). Previous studies have revealed that in a variety of cancer types, the inhibition of Homeobox genes by hypermethylation of their promoters contributes to tumorigenesis (46). Furthermore, a recent study by Kikuchi et al (47) demonstrated that HOP homeobox promoter methylation independently predicts poor prognosis in human epidermal growth factor receptor 2-negative breast cancer. These results highlighted the clinical value of Homeobox gene epigenetic silencing, and the requirement for future investigations into the clinical value of EMX2 epigenetic silencing. 


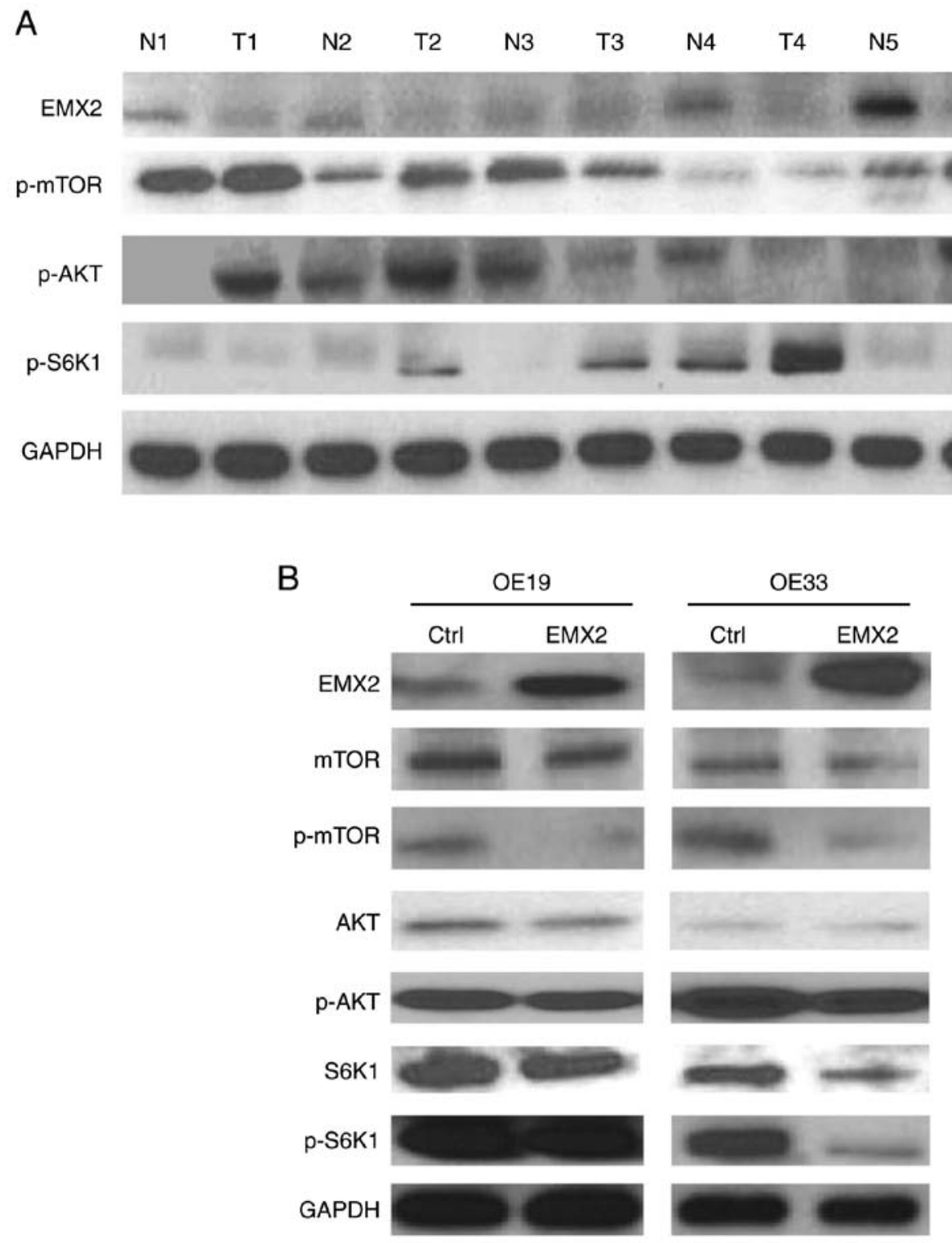

Figure 4. EMX2 inhibits EMT by suppressing AKT/mTOR/S6K1 signaling in EAC. (A) Western blot analysis of EMX2 and AKT signaling pathway proteins (p-mTOR, p-AKT and p-S6K1) in matched pairs of N and T tissues. GAPDH was used as a loading control. (B) Western blot analysis of EMX2 and AKT signaling pathway proteins (mTOR, AKT, S6K1, and p-mTOR, p-AKT and p-S6K1) in OE19 and O33 cells transfected with a vector control or EMX2. EMX2, empty spiracles homeobox 2; EMT, epithelial-mesenchymal transition; EAC, esophageal adenocarcinoma; p, phosphorylated; N, normal; T, tumor.

The PI3K/AKT and mTOR signaling pathways are crucial to numerous aspects of cell growth, survival and EMT, and have been implicated in tumor cell motility, invasion and metastasis (48-51). For instance, AKT activation was revealed to induce EMT in human oral squamous cell carcinomas (52), and subsequently it was revealed that AKT induced EMT by activating NF- $\kappa B$ (53). Conversely, the inhibition of AKT activity reversed EMT in these cells (54). AKT genes are overexpressed in EAC, and their expression has been associated with the degree of pathologic response during neoadjuvant therapy for EAC (44). S6K1 is positively regulated by mTOR, and the activation of mTOR/S6K1 signaling has been suggested to be involved in EAC progression (55). A previous study revealed that AKT signaling proteins were upregulated in the tumor tissues of patients with EAC, and AKT inhibition reduced EMT and cell cycle activity in EAC cell lines (12). In the present study, the observation that the loss of EMX2 is correlated with increased levels of p-AKT, p-mTOR and p-S6K1 in the tumor tissues is consistent with the hypothesis that the loss of EMX2 may be associated with the upregulation of AKT signaling pathways (Fig. 4A). A limitation of the study is that due to limited amount of patient samples, the total protein levels of AKT/mTOR/S6K1 were not assessed. It is unknown whether the elevated levels of p-AKT, p-mTOR and $\mathrm{p}-\mathrm{S} 6 \mathrm{~K} 1$ were due to increased total protein levels or increased phosphorylation per se. In OE19 and OE33 cancer cell lines, the phosphorylation levels of these proteins upon EMX2 expression were decreased more prominently than the changes in their total protein levels (Fig. 4B), suggesting that the suppression of AKT/mTOR/S6K1 pathway by EMX2 was not simply due to direct inhibition of AKT/mTOR/S6K1 gene expression in these cells. These data support the notion that EMX2 inhibits EMT via inhibition of AKT/mTOR/S6K1 signaling in EAC cells. 

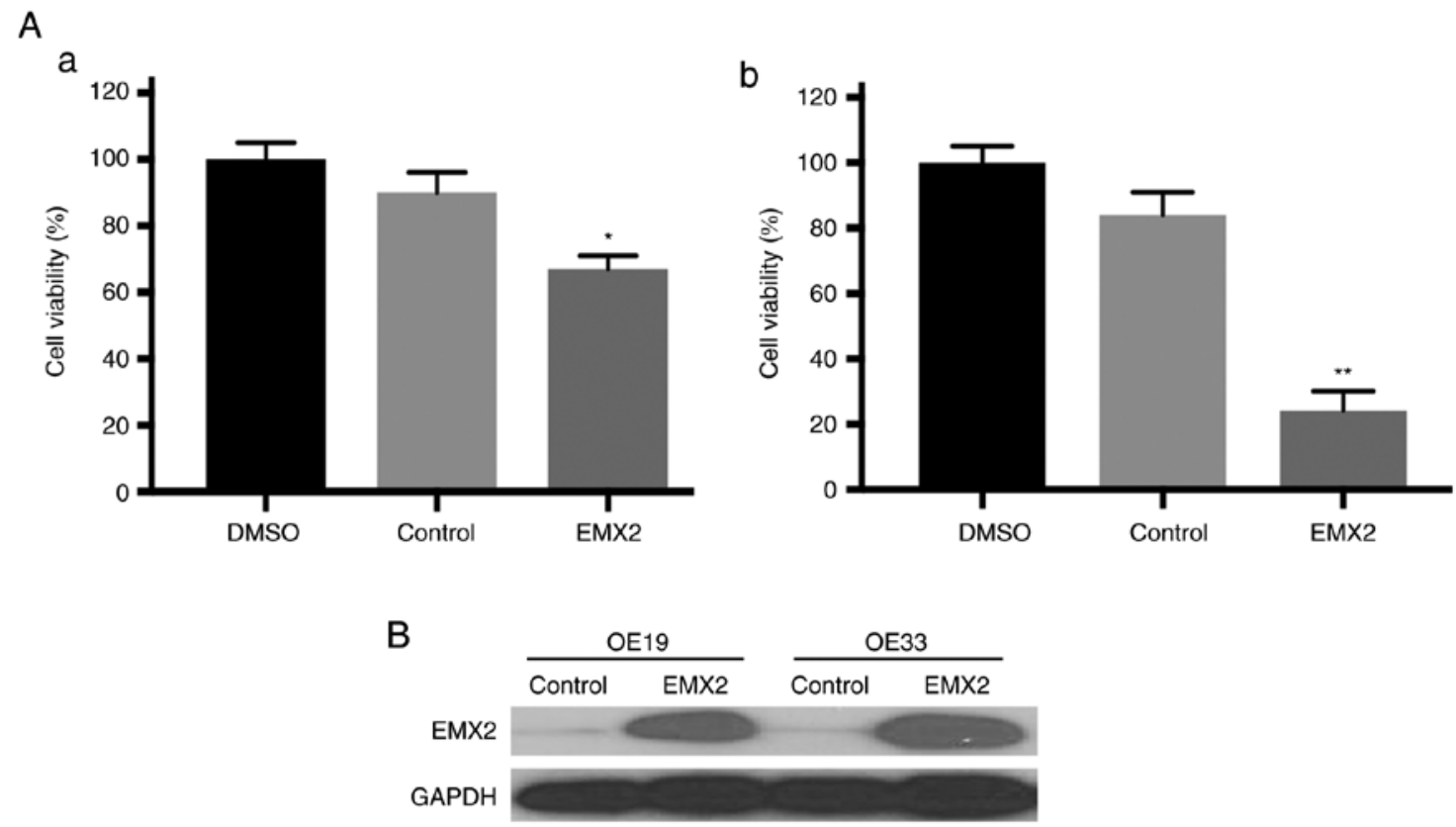

Figure 5. EMX2 sensitizes EAC cells to cisplatin. (A) Cell survival assay in (a) OE19 and (b) OE33 cells transfected with a vector control or EMX2. Cells were treated with either DMSO or cisplatin. Columns from left to right are: Vector control cells treated with DMSO, vector control cells treated with cisplatin, EMX2 transfected cells treated with cisplatin. (B) Western blot analysis of EMX2 in OE19 and OE33 cells transfected with a vector control or EMX2. GAPDH was used as a loading control. ${ }^{*} \mathrm{P}<0.05$ and ${ }^{* *} \mathrm{P}<0.01$. EMX2, empty spiracles homeobox 2 ; EAC, esophageal adenocarcinoma.

$\mathrm{AKT} / \mathrm{mTOR} / \mathrm{S} 6 \mathrm{~K} 1$ is a major hub of signal transduction pathways, and is interconnected with multiple regulatory factors. Since EMX2 is a homeobox protein, it is speculated that it may function as a specific transcription factor to regulate the expression of genes upstream of the AKT/mTOR/S6K1 pathway. Recently, the induction of EMT by GLI activation was reported in colon (56) and breast cancer (57). A previous study revealed that GLI is upregulated and is correlated with EMT and the activation of AKT pathway markers both in EAC cell lines and tissue samples (12). These studies indicated that AKT and GLI activation may be a general mechanism in EMT. Currently, it is unknown whether EMX2 functions upstream or downstream of GLI, which is the focus of the ongoing research.

Previous studies investigating EMT in EAC have revealed that the reduced expression of the E-cadherin-catenin complex, and upregulation of AKT genes in EAC, is correlated with poor prognosis $(43,44)$. The results of the present study demonstrated that EMX2 induced the upregulation of E-cadherin and inhibited AKT signaling in OE19 and OE33 cells. Furthermore, the downregulation of EMX2 in lung cancer and colorectal cancer has been associated with decreased overall survival $(22,24,28)$. Therefore, it can be suggested that the downregulation of EMX2 may be associated with a worse EAC prognosis. The manipulation of the EMX2 pathway could be used to prevent EAC progression. It may take years for this goal to be reached, however, two recent studies have made this more feasible. A previous study demonstrated that adenoviral EMX2 delivery in gastric cancer suppressed cancer cell proliferation and improved overall survival in vivo (26). Falcone et al reported that EMX2 overexpression exhibited antioncogenic activity and induced the collapse of glioblastoma cells in vitro and in vivo (58). Recent studies have also indicated an unexpected role of EMT in cancer chemoresistance (59-61). A previous study demonstrating that the knockdown of EMX2 expression in lung squamous cell carcinomas cells promoted chemoresistance (25), and the results of the present study revealing that EMX2 overexpression sensitized EAC cell lines to the chemotoxic reagent cisplatin, are consistent with these data. These observations indicate that the role of EMX2 in chemotherapy sensitivity may be general in different types of cancer. At present, the study on the role of EMX2 in chemosensitivity is limited. With further investigation, EMX2 may become a novel antitumor target, synergistic with chemotherapy options, for patients with EAC.

In summary, the present study revealed that EMX2 is epigenetically silenced in EAC, and a strong association exists between EMX2 and EMT, apoptotic and cell cycle markers and the AKT/mTOR/S6K1 pathway in EAC. These findings suggest that silencing EMX2 may prove critical in promoting cell survival, metastasis and resistance to chemotherapy. Restoration of EMX2 expression via the alleviation of epigenetic silencing may serve as a potential therapeutic strategy for the treatment of human EAC. With the advances in pharmaceutical development of safe and potent epigenetic regulatory reagents, this potential should be investigated in the future.

\section{Acknowledgements}

The authors acknowledge the important contributions of the other members of the University of California, San Francisco Thoracic Oncology Program and the research team of Dr Biao He, including Fleur Leguay, B.S.; Ngoc Hoang, B.S.; and Luis Acevedo, B.S.

\section{Funding}

The present study was supported by the Eileen D. Ludwig Endowed Fund for Thoracic Oncology Research (to BH); the Science and Technology Support Project of Hebei Province 
(132077127D to LW), the Fund for International Scientific and Technological Cooperation in Hebei Province (18397771D to LW), the Fund for Scientific and Technological Activities of Overseas Scholars by Hebei Provincial Office of Human and Social Affairs (CY201613 to LW); the Science and Technology Planning Project of Zhejiang Province Science and Technology Department (2018C37091 to YH), and the Zhejiang Provincial Natural Science Foundation of China (LY15H160048 to FD), the Jiaxing Science and Technology Project (2015BZ12001 to FD) and the Jiaxing Nanhu District Science and Technology Project (2018QC03 to FD).

\section{Availability of data and materials}

The materials and datasets used in this study are available upon request.

\section{Authors' contributions}

JJ performed cell culture, western blot and data analysis, and drafted the manuscript. LW, YZ, ZT and YH carried out western blot, qRT-PCR, cell viability, wound healing, and Transwell invasion assays. LW and $\mathrm{YH}$ also conducted data and statistical analyses and helped to draft the manuscript. $\mathrm{BH}$, YH and FD conceived of the study, participated in its design and coordination, and drafted the manuscript. All authors read and approved the final manuscript and agree to be to be accountable for all aspects of the research in ensuring that the accuracy or integrity of any part of the work are appropriately investigated and resolved.

\section{Ethical approval and consent to participate}

The present study was conducted in accordance with the ethical standards of the Declaration of Helsinki, as well as national and international guidelines, with the approval by the institutional review board of the University of California, San Francisco (UCSF). Studies involving patient tissues were approved by the Committee on Human Research (CHR approval number: H8714-11647-10) at UCSF, and written, informed consent was obtained for each patient prior to tissue specimen collection.

\section{Patient consent for publication}

Not applicable.

\section{Competing interests}

The authors declare that they have no competing interests.

\section{References}

1. Ferlay J, Soerjomataram I, Dikshit R, Eser S, Mathers C, Rebelo M, Parkin DM, Forman D and Bray F: Cancer incidence and mortality worldwide: Sources, methods and major patterns in GLOBOCAN 2012. Int J Cancer 136: E359-E386, 2015.

2. Cancer Genome Atlas Research Network; Analysis Working Group: Asan University; BC Cancer Agency; Brigham and Women's Hospital; Broad Institute; Brown University; Case Western Reserve University; Dana-Farber Cancer Institute Duke University; Greater Poland Cancer Centre, et al: Integrated genomic characterization of oesophageal carcinoma. Nature 541: 169-175, 2017.
3. Arnold M, Soerjomataram I, Ferlay J and Forman D: Global incidence of oesophageal cancer by histological subtype in 2012 . Gut 64: 381-387, 2015

4. Siegel RL, Miller KD and Jemal A: Cancer statistics, 2018. CA Cancer J Clin 68: 7-30, 2018

5. Lagarde SM, ten Kate FJ, Richel DJ, Offerhaus GJ and van Lanschot JJ: Molecular prognostic factors in adenocarcinoma of the esophagus and gastroesophageal junction. Ann Surg Oncol 14: 977-991, 2007.

6. Nieto MA, Huang RY, Jackson RA and Thiery JP: Emt: 2016. Cell 166: 21-45, 2016.

7. Polyak K and Weinberg RA: Transitions between epithelial and mesenchymal states: Acquisition of malignant and stem cell traits. Nat Rev Cancer 9: 265-273, 2009.

8. Thiery JP, Acloque H, Huang RY and Nieto MA: Epithelialmesenchymal transitions in development and disease. Cell 139: 871-890, 2009

9. Tsai JH and Yang J: Epithelial-mesenchymal plasticity in carcinoma metastasis. Genes Dev 27: 2192-2206, 2013.

10. Rees JR, Onwuegbusi BA, Save VE, Alderson D and Fitzgerald RC: In vivo and in vitro evidence for transforming growth factor-beta1-mediated epithelial to mesenchymal transition in esophageal adenocarcinoma. Cancer Res 66: 9583-9590, 2006.

11. Kestens C, Siersema PD, Offerhaus GJ and van Baal JW BMP4 signaling is able to induce an epithelial-mesenchymal transition-like phenotype in Barrett's esophagus and esophageal adenocarcinoma through induction of SNAIL2. PLoS One 11: e0155754, 2016.

12. Wang L, Jin JQ, Zhou Y, Tian Z, Jablons DM and He B: Gli is activated and promotes epithelial-mesenchymal transition in human esophageal adenocarcinoma. Oncotarget 9: 853-865, 2017.

13. Jethwa P, Naqvi M, Hardy RG, Hotchin NA, Roberts S, Spychal R and Tselepis C: Overexpression of slug is associated with malignant progression of esophageal adenocarcinoma. World J Gastroenterol 14: 1044-1052, 2008.

14. Allott EH, Morine MJ, Lysaght J, McGarrigle SA, Donohoe CL, Reynolds JV, Roche HM and Pidgeon GP: Elevated tumor expression of PAI-1 and SNAI2 in obese esophageal adenocarcinoma patients and impact on prognosis. Clin Transl Gastroenterol 3: e12, 2012.

15. Abate-Shen C: Deregulated homeobox gene expression in cancer: Cause or consequence? Nat Rev Cancer 2: 777-785, 2002.

16. Cecchi C: Emx2: A gene responsible for cortical development, regionalization and area specification. Gene 291: 1-9, 2002.

17. Brancaccio M, Pivetta C, Granzotto M, Filippis C and Mallamaci A: Emx2 and Foxg1 inhibit gliogenesis and promote neuronogenesis. Stem Cells 28: 1206-1218, 2010.

18. Falcone C, Filippis C, Granzotto $M$ and Mallamaci A: Emx2 expression levels in NSCs modulate astrogenesis rates by regulating EgfR and Fgf9. Glia 63: 412-422, 2015.

19. Holley M, Rhodes C, Kneebone A, Herde MK, Fleming M and Steel KP: Emx2 and early hair cell development in the mouse inner ear. Dev Biol 340: 547-556, 2010.

20. Jiang T, Kindt K and Wu DK: Transcription factor Emx 2 controls stereociliary bundle orientation of sensory hair cells. Elife 6 : e23661, 2017.

21. Taylor HS and Fei X: Emx2 regulates mammalian reproduction by altering endometrial cell proliferation. Mol Endocrinol 19: 2839-2846, 2005.

22. Giroux Leprieur E, Hirata T, Mo M, Chen Z, Okamoto J, Clement G, Li H, Wislez M, Jablons DM and He B: The homeobox gene EMX2 is a prognostic and predictive marker in malignant pleural mesothelioma. Lung Cancer 85: 465-471, 2014.

23. Okamoto J, Hirata T, Chen Z, Zhou HM, Mikami I, Li H, Yagui-Beltran A, Johansson M, Coussens LM, Clement G, et al: EMX2 is epigenetically silenced and suppresses growth in human lung cancer. Oncogene 29: 5969-5975, 2010.

24. Okamoto J, Kratz JR, Hirata T, Mikami I, Raz D, Segal M, Chen Z, Zhou HM, Pham P, Li H, et al: Downregulation of EMX2 is associated with clinical outcomes in lung adenocarcinoma patients. Clin Lung Cancer 12: 237-244, 2011.

25. Yue D, Li H, Che J, Zhang Y, Tolani B, Mo M, Zhang H, Zheng Q, Yang Y, Cheng R, et al: EMX2 is a predictive marker for adjuvant chemotherapy in lung squamous cell carcinomas. PLoS One 10: $\mathrm{e} 0132134,2015$.

26. Li J, Mo M, Chen Z, Chen Z, Sheng Q, Mu H, Zhang F, Zhang Y, $\mathrm{Zhi} \mathrm{XY}, \mathrm{Li} \mathrm{H}$, et al: Adenoviral delivery of the EMX2 gene suppresses growth in human gastric cancer. PLoS One 7: e45970, 2012. 
27. Qiu H, Yan Q, Luo X, Zhang H, Bao W and Wan X: EMX2 is downregulated in endometrial cancer and correlated with tumor progression. Int J Gynecol Pathol 32: 193-198, 2013.

28. Aykut B, Ochs M, Radhakrishnan P, Brill A, Höcker H, Schwarz S, Weissinger D, Kehm R, Kulu Y, Ulrich A and Schneider M: EMX2 gene expression predicts liver metastasis and survival in colorectal cancer. BMC Cancer 17: 555, 2017.

29. Zhang Y, Cao G, Yuan QG, Li JH and Yang WB: Empty spiracles homeobox 2 (EMX2) inhibits the invasion and tumorigenesis in colorectal cancer cells. Oncol Res 25: 537-544, 2017.

30. Livak KJ and Schmittgen TD: Analysis of relative gene expression data using real-time quantitative PCR and the 2(-Delta Delta C(T)) method. Methods 25: 402-408, 2001.

31. Raman V, Tamori A, Vali M, Zeller K, Korz D and Sukumar S: HOXA5 regulates expression of the progesterone receptor. J Biol Chem 275: 26551-26555, 2000.

32. Lind GE, Skotheim RI, Fraga MF, Abeler VM, Esteller M and Lothe RA: Novel epigenetically deregulated genes in testicular cancer include homeobox genes and SCGB3A1 (HIN-1). J Pathol 210: 441-449, 2006.

33. Galm O and Herman JG: Methylation-specific polymerase chain reaction. Methods Mol Med 113: 279-291, 2005.

34. Andrews PD, Knatko E, Moore WJ and Swedlow JR: Mitotic mechanics: The auroras come into view. Curr Opin Cell Biol 15 672-683, 2003

35. Gavriilidis P, Giakoustidis A and Giakoustidis D: Aurora kinases and potential medical applications of aurora kinase inhibitors: A review. J Clin Med Res 7: 742-751, 2015.

36. Goldenson B and Crispino JD: The aurora kinases in cell cycle and leukemia. Oncogene 34: 537-545, 2015

37. Kirkin V, Joos S and Zornig M: The role of Bcl-2 family members in tumorigenesis. Biochim Biophys Acta 1644: 229-249, 2004.

38. Zinkel S, Gross A and Yang E: BCL2 family in DNA damage and cell cycle control. Cell Death Differ 13: 1351-1359, 2006.

39. Alao JP: The regulation of cyclin D1 degradation: Roles in cancer development and the potential for therapeutic invention. Mol Cancer 6: 24, 2007.

40. Musgrove EA, Caldon CE, Barraclough J, Stone A and Sutherland RL: Cyclin D as a therapeutic target in cancer. Nat Rev Cancer 11: 558-572, 2011.

41. O'Brien DI, Nally K, Kelly RG, O'Connor TM, Shanahan F and $\mathrm{O}^{\prime}$ Connell J: Targeting the Fas/Fas ligand pathway in cancer. Expert Opin Ther Targets 9: 1031-1044, 2005.

42. Peter ME, Hadji A, Murmann AE, Brockway S, Putzbach W, Pattanayak A and Ceppi P: The role of CD95 and CD95 ligand in cancer. Cell Death Differ 22: 885-886, 2015.

43. Krishnadath KK, Tilanus HW, van Blankenstein M, Hop WC, Kremers ED, Dinjens WN and Bosman FT: Reduced expression of the cadherin-catenin complex in oesophageal adenocarcinoma correlates with poor prognosis. J Pathol 182: 331-338, 1997.

44. Saeed N, Shridhar R, Hoffe S, Almhanna K and Meredith KL: AKT expression is associated with degree of pathologic response in adenocarcinoma of the esophagus treated with neoadjuvant therapy. J Gastrointest Oncol 7: 158-165, 2016.

45. Rubenstein JH and Shaheen NJ: Epidemiology, diagnosis, and management of esophagealadenocarcinoma. Gastroenterology 149: 302-317 e301, 2015.

46. Rodrigues MF, Esteves CM, Xavier FC and Nunes FD: Methylation status of homeobox genes in common human cancers. Genomics 108: 185-193, 2016.
47. Kikuchi M, Katoh H, Waraya M, Tanaka Y, Ishii S, Tanaka T, Nishizawa N, Yokoi K, Minatani N, Ema A, et al: Epigenetic silencing of HOPX contributes to cancer aggressiveness in breast cancer. Cancer Lett 384: 70-78, 2017.

48. Polivka J Jr and Janku F: Molecular targets for cancer therapy in the PI3K/AKT/mTOR pathway. Pharmacol Ther 142: 164-175, 2014.

49. Saxton RA and Sabatini DM: mTOR signaling in growth, metabolism, and disease. Cell 169: 361-371, 2017.

50. Zarogoulidis P, Lampaki S, Turner JF, Huang H, Kakolyris S, Syrigos K and Zarogoulidis K: mTOR pathway: A current, up-to-date mini-review (Review). Oncol Lett 8: 2367-2370, 2014.

51. Zhou H and Huang S: Role of mTOR signaling in tumor cell motility, invasion and metastasis. Curr Protein Pept Sci 12: 30-42, 2011.

52. Grille SJ, Bellacosa A, Upson J, Klein-Szanto AJ, van Roy F, Lee-Kwon W, Donowitz M, Tsichlis PN and Larue L: The protein kinase Akt induces epithelial mesenchymal transition and promotes enhanced motility and invasiveness of squamous cell carcinoma lines. Cancer Res 63: 2172-2178, 2003.

53. Julien S, Puig I, Caretti E, Bonaventure J, Nelles L, van Roy F, Dargemont C, de Herreros AG, Bellacosa A and Larue L: Activation of NF-kappaB by Akt upregulates Snail expression and induces epithelium mesenchyme transition. Oncogene 26: 7445-7456, 2007

54. Hong KO, Kim JH, Hong JS, Yoon HJ, Lee JI, Hong SP and Hong SD: Inhibition of Akt activity induces the mesenchymal-to-epithelial reverting transition with restoring E-cadherin expression in KB and KOSCC-25B oral squamous cell carcinoma cells. J Exp Clin Cancer Res 28: 28, 2009.

55. Wang Y, Ding Q, Yen CJ, Xia W, Izzo JG, Lang JY, Li CW, Hsu JL, Miller SA, Wang X, et al: The crosstalk of mTOR/S6K1 and hedgehog pathways. Cancer Cell 21: 374-387, 2012.

56. Varnat F, Duquet A, Malerba M, Zbinden M, Mas C, Gervaz P, Ruiz I and Altaba A: Human colon cancer epithelial cells harbour active HEDGEHOG-GLI signalling that is essential for tumour growth, recurrence, metastasis and stem cell survival and expansion. EMBO Mol Med 1: 338-351, 2009.

57. Neelakantan D, Zhou H, Oliphant MUJ, Zhang X, Simon LM, Henke DM, Shaw CA, Wu MF, Hilsenbeck SG, White LD, et al: EMT cells increase breast cancer metastasis via paracrine GLI activation in neighbouring tumour cells. Nat Commun 8: 15773, 2017.

58. Falcone C, Daga A, Leanza G and Mallamaci A: Emx2 as a novel tool to suppress glioblastoma. Oncotarget 7: 41005-41016, 2016.

59. Fischer KR, Durrans A, Lee S, Sheng J, Li F, Wong ST, Choi H, El Rayes T, Ryu S, Troeger J, et al: Epithelial-to-mesenchymal transition is not required for lung metastasis but contributes to chemoresistance. Nature 527: 472-476, 2015.

60. Wang J, Wei Q, Wang X, Tang S, Liu H, Zhang F, Mohammed MK, Huang J, Guo D, Lu M, et al: Transition to resistance: An unexpected role of the EMT in cancer chemoresistance. Genes Dis 3: 3-6, 2016

61. Zheng X, Carstens JL, Kim J, Scheible M, Kaye J, Sugimoto H, Wu CC, LeBleu VS and Kalluri R: Epithelial-to-mesenchymal transition is dispensable for metastasis but induces chemoresistance in pancreatic cancer. Nature 527: 525-530, 2015. 